\title{
Uniform-acceptance force-bias Monte Carlo method with time scale to study solid-state diffusion
}

\author{
Maarten J. Mees \\ Department of Physics, University of Leuven, Celestijnenlaan 200 D, B-3001 Leuven, Belgium and \\ imec, Kapeldreef 75, B-3001 Leuven, Belgium \\ Geoffrey Pourtois* \\ imec, Kapeldreef 75, B-3001 Leuven, Belgium and Department of Chemistry, University of Antwerp, \\ Universiteitsplein 1, B-2610 Wilrijk-Antwerp, Belgium \\ Erik C. Neyts \\ Department of Chemistry, University of Antwerp, Universiteitsplein 1, B-2610 Wilrijk-Antwerp, Belgium \\ Barend J. Thijsse \\ Department of Materials Science and Engineering, Delft University of Technology, Mekelweg 2, 2628 CD Delft, The Netherlands \\ André Stesmans \\ Department of Physics, University of Leuven, Celestijnenlaan 200 D, B-3001 Leuven, Belgium \\ (Received 22 November 2011; revised manuscript received 6 March 2012; published 3 April 2012)
}

\begin{abstract}
Monte Carlo (MC) methods have a long-standing history as partners of molecular dynamics (MD) to simulate the evolution of materials at the atomic scale. Among these techniques, the uniform-acceptance force-bias Monte Carlo (UFMC) method [G. Dereli, Mol. Simul. 8, 351 (1992)] has recently attracted attention [M. Timonova et al., Phys. Rev. B 81, 144107 (2010)] thanks to its apparent capacity of being able to simulate physical processes in a reduced number of iterations compared to classical MD methods. The origin of this efficiency remains, however, unclear. In this work we derive a UFMC method starting from basic thermodynamic principles, which leads to an intuitive and unambiguous formalism. The approach includes a statistically relevant time step per Monte Carlo iteration, showing a significant speed-up compared to MD simulations. This time-stamped force-bias Monte Carlo (tfMC) formalism is tested on both simple one-dimensional and three-dimensional systems. Both test-cases give excellent results in agreement with analytical solutions and literature reports. The inclusion of a time scale, the simplicity of the method, and the enhancement of the time step compared to classical MD methods make this method very appealing for studying the dynamics of many-particle systems.
\end{abstract}

DOI: 10.1103/PhysRevB.85.134301 PACS number(s): 02.70.Ns, 02.70.Tt, 02.50.Ng, 68.35.Fx

\section{INTRODUCTION}

The development of fundamental understanding in material science starts at the atomic level, where concerted movements set the properties of a material and its reaction upon the application of external factors. With the current development of nanostructured materials, gaining insights into the dynamics that drive the evolution of a system along physically representative time windows is becoming a problem of prime importance.

In that respect, molecular dynamics (MD) has long proved to be a very robust and useful technique that offers unique insights into the dynamical behavior of a system. However, a large gap is often observed between the events captured by the MD simulations and the experimental ones, mainly due to the fact that monitoring the evolution of a material between different equilibrium states is a computationally very demanding task. Aside from the limitations bound to the physical dimensions of the system, the time window of the events that can be assessed is relatively limited and typically evolves in the range of picoseconds (ps) to nanoseconds (ns). Extending the limits of the simulated time window to reach different equilibrium states, while keeping access to the dynamics of the evolution, is hence becoming a very intensive field of research. ${ }^{1,2}$ Much pioneering work has already been done to bridge the gap, ${ }^{3-10}$ in which long-time atomic events are accessed through MD simulations. Among the different approaches proposed in literature, a very popular one consists of modifying the potential-energy surface with a bias potential that goes to zero near transition states. ${ }^{3,4}$ This enhances all transition rates of reactions, while preserving the relative rates. Although these techniques offer very interesting insights, further improvements are still (highly) desirable. ${ }^{1,2}$

Alternatively, a set of techniques derived from stochastic approaches and based on atomic forces ${ }^{11-15}$ might open the way toward further developments. Although these force-bias Monte-Carlo (fbMC) methods have been little used in the past (see for instance Refs. 16 and 17) they recently regained attention through the works of Timonova ${ }^{18}$ and Neyts et al. ${ }^{19,20}$ Contrary to MD algorithms, fbMC relies on a force-bias probabilistic description of the atomic motion. This approach recently has been shown to be successfully able to reproduce long-term events that take place in materials upon phase transition, surface diffusion, and growth. ${ }^{17-20}$ The simplicity of the fbMC approach is not its only attractiveness, since, compared to a classical MD approach, it has been reported to require a reduced number of numerical iterations to access physical meaningful results. ${ }^{18}$ Aside from this apparent gain in efficiency, a clear quantitative comparison between the MD 
and fbMC approaches turns out to be a difficult task due to the stochastic roots of fbMC and its lack of a time scale. As a consequence, the possible origin of the apparent efficiency of fbMC with respect to classical MD technique remains incompletely understood.

Through this article, we aim to fill this gap by building a rigorous derivation, starting from the canonical ensemble, of a force-bias Monte Carlo formalism. Although the obtained formalism differs slightly from the original one proposed by Dereli (uniform-acceptance force-bias Monte Carlo, UFMC), ${ }^{15,18}$ it offers the advantage of providing an intuitive and solid derivation for the technique. The comparison between our time-stamped force-bias Monte Carlo (tfMC) and the MD formalism is carried out through the formulation of a statistically relevant time bound to the atomic displacement. It is found that the effective gain in time per tfMC iteration is in many cases larger than the "standard" 1 fs time step used in classical MD calculations.

The paper is organized as follows. First, we provide a detailed derivation of the tfMC approach and illustrate its working principles using a simple one-dimensional idealized system (Sec. II). We then demonstrate the relation between the atomic motion and a statistically relevant time definition (Sec. III). Note that, for the convenience of the reader, we remained consistent with the notation used in the prior paper of Timonova et al. ${ }^{18}$

\section{DERIVATION OF THE METHOD}

This section is structured as follows: starting from the principles of a canonical ensemble, Sec. II A derives the tfMC method. A more intuitive idea of the algorithm is sketched in Sec. II B, followed by a proof of these ideas in Sec. II C. Eventually, Sec. IID discusses time evolution in the MC algorithm, allowing for a quantitative comparison with MD.

\section{A. The time-stamped force-bias MC algorithm}

As pointed-out by Timonova et al., ${ }^{18}$ the essence of the force-bias Monte Carlo approach relies on an iterative modification of the atomic positions in which only their atomic forces are accounted for. To be valid, such a stochastic displacement of the particles has to remain statistically consistent with the evolution of the system. In a canonical ensemble at temperature $T$, containing $N$ particles and with a Hamiltonian $H$, the probability density function $\rho$ that describes the system is given by

$$
\rho=\frac{\exp (-\beta H)}{\int \exp (-\beta H) d \Gamma},
$$

with $\Gamma$ representing the phase space in which all the possible states of the particles are described and $\beta=\left(k_{B} T\right)^{-1}$. The Hamiltonian of the $N$ interacting particles associated with masses $m_{i}$ can take the form

$$
H=\sum_{i=1}^{N} \frac{\boldsymbol{p}_{i}^{2}}{2 m_{i}}+U\left(\boldsymbol{x}_{1}, \boldsymbol{x}_{2}, \ldots, \boldsymbol{x}_{N}\right),
$$

where $\boldsymbol{p}_{i}$ is the momentum vector of the particle $i$ and the potential $U$ accounts for both the particle's interaction and the presence of a possible external applied potential. During its evolution, the system can adopt a state associated with a different set of coordinates such as $\boldsymbol{x}^{*}=\left(\boldsymbol{x}_{1}^{*}, \boldsymbol{x}_{2}^{*}, \ldots, \boldsymbol{x}_{N}^{*}\right)$, where a reformulation of $U$ based on a Taylor expansion in the neighborhood of this state leads to

$$
\begin{aligned}
U(\boldsymbol{x}) & =U\left(\boldsymbol{x}^{*}\right)+(\mathrm{d} U)\left(\boldsymbol{x}^{*}\right) \circ\left(\boldsymbol{x}-\boldsymbol{x}^{*}\right)^{T}+\mathcal{O}\left(\boldsymbol{x}^{2}\right) \\
& =U\left(\boldsymbol{x}^{*}\right)-\sum_{i=1}^{N} \boldsymbol{F}_{i} \cdot\left(\boldsymbol{x}_{i}-\boldsymbol{x}_{i}^{*}\right)+\mathcal{O}\left(\boldsymbol{x}^{2}\right),
\end{aligned}
$$

with $\boldsymbol{F}_{i}$ being the force acting on particle $i$. If the Taylor expansion is truncated to its first-order term, meaning that $\mathcal{O}\left(\boldsymbol{x}^{2}\right)$ is negligible for all $x_{i, j} \in\left[x_{i, j}^{*}-\Delta / 2, x_{i, j}^{*}+\Delta / 2\right]$, with $\Delta$ being the range of atomic displacement for which the expansion is valid and $j$ being the $x, y$, and $z$ directions, then the Hamiltonian in the neighborhood of $\boldsymbol{x}^{*}$ takes the form

$$
\widetilde{H}=U\left(x^{*}\right)+\sum_{i=1}^{N} \sum_{j=1}^{3}\left(\frac{p_{i, j}^{2}}{2 m_{i}}-F_{i, j}\left(x_{i, j}-x_{i, j}^{*}\right)\right) .
$$

By combining Eqs. (1) and (4), the probability density function $\tilde{\rho}$ in coordination space in the neighborhood of $\boldsymbol{x}^{*}$ takes the form

$$
\tilde{\rho}=\exp \left[-\beta U\left(\boldsymbol{x}^{*}\right)\right] \frac{\prod_{i, j} \exp \left[\beta F_{i, j}\left(x_{i, j}-x_{i, j}^{*}\right)\right]}{\int_{\mathcal{V}} \exp [-\beta U(\boldsymbol{x})] d \boldsymbol{x}} .
$$

Equation (5) will be used to derive the necessary probability function used to drive the tfMC algorithm.

Before going into the details of this derivation, it is useful to gain some insights about the MC-algorithm mechanics. We assume that at a given time the particles are located in the (small) coordinate space intervals $\left(\left[x_{i, j}^{*}-\Delta / 2, x_{i, j}^{*}+\right.\right.$ $\Delta / 2])_{i, j}$ which we label as a volume $\mathcal{V}_{i}$ given by

$$
\mathcal{V}_{i}=\bigcup_{i, j}\left[x_{i, j}^{*}-\Delta / 2, x_{i, j}^{*}+\Delta / 2\right] .
$$

Note that this volume corresponds to the one described by $\tilde{\rho}$. Suppose now that, during a short but unspecified time, the particles are allowed to move in this volume $\mathcal{V}_{i}$. The question arises of being able to identify the probability that, during this time interval, the particles move to another volume $\mathcal{V}_{f}$ which has the same form as $\mathcal{V}_{i}$, but which is shifted with respect to $\boldsymbol{x}^{*}$. Assuming that this probability can be quantified, all the necessary ingredients to use a MC algorithm would be present to choose the next volume $\mathcal{V}_{f}$. The latter will then become the new initial volume $\mathcal{V}_{i}$ bound to a new coordinate $\boldsymbol{x}^{*}$. Upon the repetition of the procedure, successive volumes are generated in which all particles are captured and the dynamics of the system is described (see Sec. II B).

For such an approach to be operational, one naturally needs to develop an explicit formulation for the probability of going from $\mathcal{V}_{i}$ to $\mathcal{V}_{f}$. It turns out that the concept of a conditional probability is perfectly suited for this task. The conditional probability of going from $\mathcal{V}_{i}$ to $\mathcal{V}_{f}$ is defined as

$$
P\left(\text { state } \mathcal{V}_{f} \mid \text { state } \mathcal{V}_{i}\right)=\frac{P\left(\left(x \in \mathcal{V}_{i}\right) \cap\left(x \in \mathcal{V}_{f}\right)\right)}{P\left(x \in \mathcal{V}_{i}\right)}
$$

Using to our advantage the fact that $P(\boldsymbol{x} \in \mathcal{A})=\int_{\mathcal{A}} \rho(\boldsymbol{x}) d \boldsymbol{x}$ and that $\rho=\widetilde{\rho}$ on $\mathcal{V}_{i}$, the conditional probability can be 
reformulated as

$$
\begin{aligned}
P\left(\text { state } \mathcal{V}_{f} \mid \text { state } \mathcal{V}_{i}\right) & =\frac{\int_{\mathcal{V}_{i} \cap \mathcal{V}_{f}} \rho(\boldsymbol{x}) d \boldsymbol{x}}{\int_{\mathcal{V}_{i}} \rho(\boldsymbol{x}) d \boldsymbol{x}} \\
& =\frac{\int_{\mathcal{V}_{i} \cap \mathcal{V}_{f}} \widetilde{\rho}(\boldsymbol{x}) d \boldsymbol{x}}{\int_{\mathcal{V}_{i}} \widetilde{\rho}(\boldsymbol{x}) d \boldsymbol{x}} \\
& =\frac{\prod_{i, j} \int_{\mathcal{V}_{i} \cap \mathcal{V}_{f}} e^{\beta F_{i, j}\left(x_{i, j}-x_{i, j}^{*}\right)} d \boldsymbol{x}}{\prod_{i, j} \int_{\mathcal{V}_{i}} e^{\beta F_{i, j}\left(x_{i, j}-x_{i, j}^{*}\right)} d \boldsymbol{x}} .
\end{aligned}
$$

For notational reasons we define $\gamma_{i, j}$ as

$$
\gamma_{i, j}=\frac{F_{i, j} \Delta}{2 k_{B} T}
$$

and write the coordinate $\boldsymbol{x}_{f}^{*}$ of the final volume on the basis of its initial coordinate $\boldsymbol{x}_{i}^{*}$ through a translation vector $\Delta \boldsymbol{\xi}$ as

$$
x_{f}^{*}=x_{i}^{*}+\Delta \xi,
$$

with $\xi$ being a vector defined as $\xi=\left(\xi_{1,1}, \xi_{1,2}, \ldots, \xi_{N, 3}\right)$, where each component $\xi_{i, j}$ takes values in $[-1,1]$ and with $\Delta$ corresponding to the maximal displacement of a particle [see Eq. (6)]. Equations (9) and (10) allow us to rewrite Eq. (8) as $P\left(\right.$ state $\mathcal{V}_{f} \mid$ state $\left.\mathcal{V}_{i}\right)=\prod_{i, j} P_{i, j}\left(\xi_{i, j}\right)$ with $P_{i, j}\left(\xi_{i, j}\right)$ given by

$$
P_{i, j}\left(\xi_{i, j}\right)= \begin{cases}\frac{e^{\gamma_{i, j}\left(2 \xi_{i, j}+1\right)}-e^{-\gamma_{i, j}}}{e^{\gamma_{i, j}}-e^{-\gamma_{i, j}}}, & \xi_{i, j} \in[-1,0[, \\ \frac{e^{\gamma_{i, j}}-e^{\gamma_{i, j}\left(2 \xi_{i, j}-1\right)}}{e^{\gamma_{i, j}}-e^{-\gamma_{i, j}}}, & \left.\left.\xi_{i, j} \in\right] 0,1\right] .\end{cases}
$$

Equation (11) constitutes the basic ingredient of our tfMC algorithm and defines the location of the particles in coordination space throughout the iterations.

In practice, for each step, a different random coordinate pair $\left(\xi_{0}, P_{0}\right)$ is chosen with $\xi_{0} \in[-1,1]$ and $P_{0} \in[0,1]$ for all the particles in all the directions. The next interval of particle $i$ in the direction $j$ will be around $x_{i, j}^{*}+\xi_{0} \Delta$ when $P_{i, j}\left(\xi_{0}\right)>P_{0}$. If $P_{i, j}\left(\xi_{0}\right)<P_{0}$, a new random pair $\left(\xi_{0}, P_{0}\right)$ is generated for that degree of freedom and the acceptance is checked again. This process consists of examining where the sampled pair $\left(\xi_{0}, P_{0}\right)$ is located with respect to the curve of the conditional probability $P_{i, j}$ (see Fig. 1). When it falls below the conditional

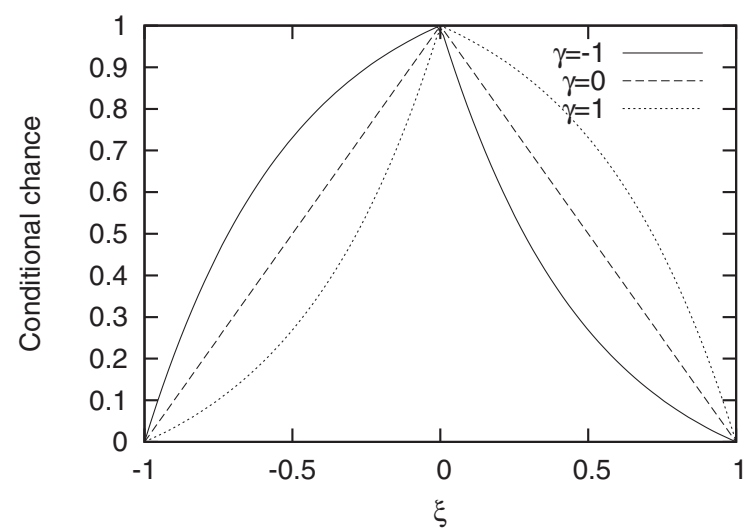

FIG. 1. The function $P_{i, j}$ for different $\gamma$ values [see Eq. (9)]. curve, the new position of the interval, defined as $x_{i, j}^{*}+\xi_{0} \Delta$, is accepted. In contrast, when $\left(\xi_{0}, P_{0}\right)$ falls above the curve, a new random coordinate is generated and the evaluation is redone. Note that the generation of random coordinates (intrinsic to MC algorithms) only requires a negligible amount of time compared with the one needed to evaluate the atomic force.

The evolution of the conditional probability with respect to $\xi$ is illustrated in Fig. 1, in which the dimensionless factor $\gamma$ corresponds to Eq. (9). From this equation it follows that the sign of $\gamma$ is equal to the sign of the force $F$. Because $\xi \Delta$ is the displacement of the particle, it follows that for a positive value of the product $\gamma$ times $\xi$, the particle goes downhill (the displacement and force point in the same direction), while for negative one, the particle goes uphill (the displacement and force point in opposite directions). The combination of these considerations together with the shape of the conditional probability (Fig. 1) show that the particle has a lower probability of going uphill than downhill.

\section{B. An interpretation of the tfMC algorithm}

An important postulate of the previous section relies on the fact that the tfMC approach is able to describe the evolution of the system dynamics based on a simple probabilistic description of a transition from a given volume $\mathcal{V}_{i}$ to another one $\mathcal{V}_{f}$, independently of the velocity of the particles. This interpretation is counterintuitive and at odds with the classical MD formalism. Indeed, in MD the generated atomic positions $x_{i, j}\left(t_{n}+\tau\right)$ are determined based on the velocity of the particles $v_{i, j}\left(t_{n}\right)$ at a previous time $t_{n}$ as

$$
x_{i, j}\left(t_{n}+\tau\right)=x_{i, j}\left(t_{n}\right)+v_{i, j}\left(t_{n}\right) \tau+\frac{F_{i, j}}{m_{i}} \frac{\tau^{2}}{2},
$$

where $\tau$ is the MD time step chosen. Because there is no direct inclusion of the particle velocity in tfMC, successive steps in this method may appear to be highly unphysical. Note however that a single step in tfMC does correspond to a physical event (see Sec. II A). In this and in the following section (Sec. II C), we show that after a sufficient number of iterations, tfMC allows us to gain a lot of insight into the physics of the system due to the proper description of the density probability function. This implies that the system simulated by tfMC is mainly located in an equilibrium state and that it leaves every now and then to evolve toward another equilibrium configuration. Indeed, the probability density function dictates that the probability of finding a particle in a potential well of the potential energy surface is in general much higher than the one of finding it on a hypersurface dividing two equilibrium states.

The basic ingredients of the tfMC algorithm are the coordinates of the particles, the forces acting on them (when they are in the volume $\mathcal{V}_{i}$ ), the temperature $T$, and the maximum atomic displacement $\Delta$. $\Delta$ may not be chosen to be too large, such that the first-order Taylor expansion formulated in Eq. (3) is not violated. In order to provide some insights on the impact of the size of $\Delta$ on the correctness of the simulations, we performed a one-dimensional test as illustrated in Fig. 2 (see Sec. III A for further details). 


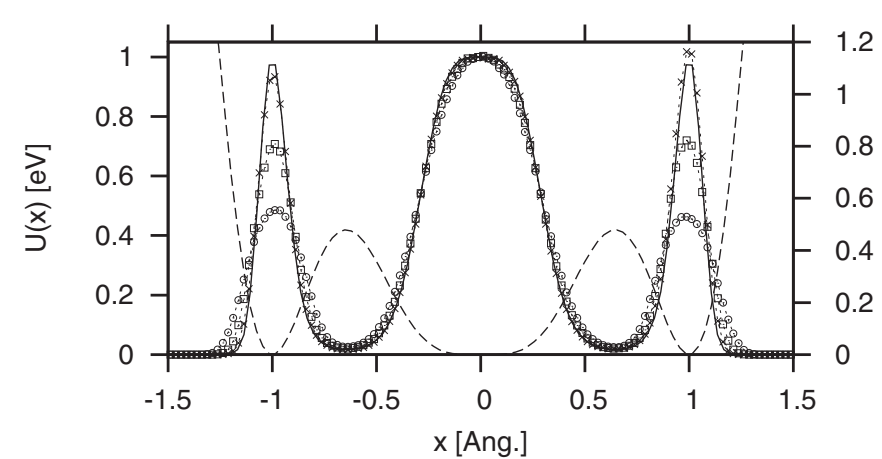

Figure 2 illustrates a simple one-particle one-dimensional potential energy surface $U(x)$ defined as $U(x)=2.5 x^{2} Q[1-$ $\cos (2 \pi x)]$ with $Q=0.25 \mathrm{eV}$, and its corresponding probability density function $f(x) \propto \exp [-\beta U(x)]$ at $T=$ $1200 \mathrm{~K}$. As expected, the probability of being in a minimum of the potential surface is higher than the one of being on a maximum, and depends on the temperature of the system. The simulated probability density function obtained for different $\Delta$ 's in Fig. 2 has been computed by accounting for the number of occurrences of the particle in a given interval divided by the number of times the particle was found to be around the origin. For $\Delta$ values larger than $0.10 \AA$, the tfMC simulation starts deviating from the analytical solution. This corresponds to the point where the potential curve requires a Taylor expansion with higher-order terms than the first-order one of Eq. (3). The results for $\Delta=0.10 \AA$ suggest that the requirement of detailed balance is respected for sufficiently small $\Delta$ 's. In the next section, we prove that this is indeed the case.

\section{Detailed balance in the tfMC algorithm}

tfMC simulates the correct probability density function of a canonical ensemble if it meets the requirement of detailed balance, i.e.,

$$
\begin{aligned}
& P\left(\text { state } \mathcal{V}^{\prime} \mid \text { state } \mathcal{V}\right) \exp (-\beta U) \\
& \quad=P\left(\text { state } \mathcal{V} \mid \text { state } \mathcal{V}^{\prime}\right) \exp \left(-\beta U^{\prime}\right) .
\end{aligned}
$$

In Eq. (13), $U$ and $U^{\prime}$ are the potential energies of the states $\mathcal{V}$ and $\mathcal{V}^{\prime}$, respectively. If $\Delta$ is sufficiently small, $U^{\prime}-U$ can be written as

$$
U^{\prime}-U \approx-\sum_{i, j} F_{i, j} \xi_{i, j} \Delta=\sum_{i, j}\left(U_{i, j}^{\prime}-U_{i, j}\right) .
$$

Substituting Eq. (14) into Eq. (13) and using the fact that $P\left(\right.$ state $\mathcal{V}^{\prime} \mid$ state $\left.\mathcal{V}\right)=\prod_{i, j} P_{i, j}\left(\xi_{i, j}\right)$ lead us to the situation that if

$$
\frac{P_{i, j}\left(\xi_{i, j}\right)}{P_{i, j}^{\prime}\left(\xi_{i, j}^{\prime}\right)}=\exp \left[-\beta\left(U_{i, j}^{\prime}-U_{i, j}\right)\right]
$$

is true for all particles $i$ in all directions $j$, the requirement of detailed balance as formulated in Eq. (13) is satisfied. Proving Eq. (15) for a particle $i$ in a direction $j$ hence ensures the detailed balance condition of Eq. (13).

For the purposes of clarity, the indices $i$ and $j$ are dropped in what follows. Note that the first-order Taylor expansion of Eq. (14) yields $F=F^{\prime}$ and hence $P=P^{\prime}$ [see Eq. (11)]. Further, it is clear that because $\xi \Delta$ represents the particles

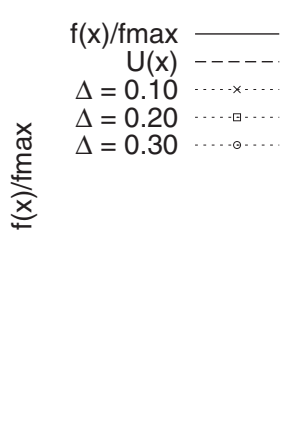

FIG. 2. The potential energy surfaces $U(x)=2.5 x^{2} Q[1-\cos (2 \pi x)] \quad($ dashed line) with $Q=0.25 \mathrm{eV}$ and the corresponding density function $f(x)$ (solid line) at $T=1200 \mathrm{~K}$. The density probability function computed using the tfMC approaches (for $\Delta$ going from 0.1 to $0.3 \AA$ ) is compared to the analytical solution.

displacement, $\xi=-\xi^{\prime}$. These considerations combined with Eq. (11) prove that tfMC complies with Eq. (15) when $\xi>0$, as

$$
\begin{aligned}
\frac{P(\xi)}{P^{\prime}\left(\xi^{\prime}\right)} & =\frac{e^{\gamma}-e^{\gamma(2 \xi-1)}}{e^{\gamma\left(2 \xi^{\prime}+1\right)}-e^{-\gamma}}=\frac{e^{\gamma}-e^{-(\beta \Delta U+\gamma)}}{e^{(\beta \Delta U+\gamma)}-e^{-\gamma}} \\
& =\frac{e^{-\beta \Delta U / 2}\left(e^{(\beta \Delta U / 2+\gamma)}-e^{-(\beta \Delta U / 2+\gamma)}\right)}{e^{\beta \Delta U / 2}\left(e^{(\beta \Delta U / 2+\gamma)}-e^{-(\beta \Delta U / 2+\gamma)}\right)} \\
& =e^{-\beta \Delta U} .
\end{aligned}
$$

When $\xi<0$, the result is analogous, which confirms that the tfMC method obeys the requirement of a detailed balance for a canonical ensemble.

The intuitive approach used in this article to derive the tfMC distinguishes it from other fbMC methods inasmuch as all input parameters have an unambiguous physical meaning. Although the work of Timonova et al. ${ }^{18}$ properly defined the potential and the limitations of fbMC techniques, we here extend the method by including the concept of time in tfMC in the following section.

\section{Time in the tfMC algorithm}

In order to quantitatively compare the tfMC with classical $\mathrm{MD}$, the notion of time should be quantified. We consider a particle $i$ moving along the $j$ direction in the interval $\left[x_{i, j}^{*}-\right.$ $\left.\Delta / 2, x_{i, j}^{*}+\Delta / 2\right]$. The force exerted on this particle in this direction in the tfMC method is given by the constant $F_{i, j}$. For a given iteration $n$, the particle $i$ moves then in the direction $j$ by a distance

$$
x_{i, j}\left(t_{n}+\Delta t_{n}\right)-x_{i, j}\left(t_{n}\right)=v_{i, j}\left(t_{n}+\Delta t_{n} / 2\right) \Delta t_{n} .
$$

Interestingly, the time step $\Delta t_{n}$ should be the same for all particles, but $\Delta t_{n}$ and $\Delta t_{m}$ do not need to be identical when $n \neq m$. This implies that, unlike MD, the notion of a constant time step along the simulation cannot be used. However, one can use the notion of an average time step $\langle\Delta t\rangle$ through the simulation as illustrated below.

Using Eq. (17) and exploiting the fact that velocities and coordinates are independent variables due to the form of the Hamiltonian used in Eq. (2), $\langle\Delta t\rangle$ can be expressed as

$$
\langle\Delta t\rangle=\frac{\left\langle\left|\Delta x_{i, j}\right|\right\rangle}{\left\langle\left|v_{i, j}\right|\right\rangle},
$$


with \langle\rangle being the expectation value. The averaged speed in Eq. (18) is easily found as

$$
\begin{aligned}
\left\langle\left|v_{i, j}\right|\right\rangle 7 & =\frac{\int_{-\infty}^{\infty}\left|p_{i j} / m_{i}\right| e^{-\beta p_{i j}^{2} /\left(2 m_{i}\right)} d p_{i j}}{\int_{-\infty}^{\infty} e^{-\beta p_{i j}^{2} /\left(2 m_{i}\right)} d p_{i j}} \\
& =\frac{\left(2 / m_{i}\right) \int_{0}^{\infty} p_{i j} e^{-\beta p_{i j}^{2} /\left(2 m_{i}\right)} d p_{i j}}{\int_{-\infty}^{\infty} e^{-\beta p_{i j}^{2} /\left(2 m_{i}\right)} d p_{i j}} \\
& =\sqrt{\frac{2 k_{B} T}{\pi m_{i}}} .
\end{aligned}
$$

Note that the averaged speed of the particle depends on both its mass and the temperature $T$ of the system. From Eq. (18) it follows that $\left\langle\left|\Delta x_{i, j}\right|\right\rangle$ has to show the same dependence to prevent generating the unphysical situation that $\langle\Delta t\rangle$ is different for particles with different masses. However, Eq. (11) shows that in its current formulation $\xi_{i, j}$ and hence $\Delta x_{i, j}$ [due to Eq. (10)], is independent of the particles mass. This problem is solved by deriving an expression for the expectation value of $\left|\xi_{i, j}\right|$ from which a constraint for $\left\langle\left|\Delta x_{i, j}\right|\right\rangle$ follows.

Using $\gamma$ again as defined in Eq. (9), the expectation value of $\left|\xi_{i, j}\right|$ can be rewritten as

$$
\begin{aligned}
\left\langle\left|\xi_{i, j}\right|\right\rangle= & \int_{-1}^{1}\left|\xi_{i, j}\right| p_{i, j}\left(\xi_{i, j}\right) d \xi_{i, j} \\
= & -\int_{-1}^{0} \xi_{i, j} \frac{e^{\gamma\left(2 \xi_{i, j}+1\right)}-e^{-\gamma}}{e^{\gamma}-e^{-\gamma}} d \xi_{i, j} \\
& +\int_{0}^{1} \xi_{i, j} \frac{e^{\gamma}-e^{\gamma\left(2 \xi_{i, j}-1\right)}}{e^{\gamma}-e^{-\gamma}} d \xi_{i, j} \\
= & \frac{1}{2}\left(1-\frac{\gamma \cosh (\gamma)-\sinh (\gamma)}{\gamma^{2} \sinh (\gamma)}\right) .
\end{aligned}
$$

In the limit $\gamma \rightarrow 0,\left\langle\left|\xi_{i, j}\right|\right\rangle$ is equal to $1 / 3$ and monotonically increases to the value $1 / 2$ for the limit $|\gamma| \rightarrow+\infty$. As a result, Eq. (20) can be rewritten in a first-order approximation as

$$
\left\langle\left|\xi_{i, j}\right|\right\rangle \approx \frac{1}{3}
$$

from which follows together with Eq. (10) that

$$
\left\langle\left|\Delta x_{i, j}\right|\right\rangle \approx \frac{\Delta}{3} .
$$

The approximation of Eq. (21) turns out to be relatively robust; during the numerical tests performed in Sec. III, $|\gamma|$ has never been observed to be larger than 2. Assuming the border case in which Eq. (20) is evaluated for $\gamma=2$ or $\gamma=-2,\left\langle\left|\xi_{i, j}\right|\right\rangle$ adopts a numerical value of $\pm(1 / 3+0.035)$, which lies within a reasonable numerical accuracy.

Equations (18) and (19) set the constraint that $\left\langle\left|\Delta x_{i, j}\right|\right\rangle$ has to have a mass dependence to obtain a relevant $\langle\Delta t\rangle$ definition.
This can be achieved by normalizing the maximum atomic displacement $\Delta_{i}$ for particle $i$ such as

$$
\Delta_{i}=\Delta \sqrt{\frac{m_{\min }}{m_{i}}},
$$

with $m_{\min }=\min \left\{m_{i} \mid i=1, \ldots, N\right\}$. This implies that the lightest element in the system considered will have the largest average displacement, and that the other elements will be associated with a smaller average perturbation than $\Delta$. Since $\left\langle\left|\Delta x_{i, j}\right|\right\rangle$ now shows a dependence on the mass, Eq. (9) is reformulated as

$$
\gamma_{i, j}=\frac{F_{i, j} \Delta_{i}}{2 k_{B} T}
$$

and the relation between $\boldsymbol{x}_{i}^{*}$ and $\boldsymbol{x}_{f}^{*}$ in Eq. (10) takes the form

$$
\boldsymbol{x}_{f}^{*}=\boldsymbol{x}_{i}^{*}+\boldsymbol{\Delta} \cdot \boldsymbol{\xi}
$$

with $\boldsymbol{\Delta}=\left(\Delta_{1}, \Delta_{1}, \Delta_{1}, \Delta_{2}, \ldots, \Delta_{N}\right)$. The averaged time step per simulation is now given by

$$
\langle\Delta t\rangle \approx \frac{\Delta}{3} \sqrt{\frac{\pi m_{\min }}{2 k_{B} T}} .
$$

For illustration purposes, we summarized the evolution of the averaged time step per iteration for a set of different temperatures and atomic masses in Table I, for a fixed value of $\Delta$. As expected from Eq. (26), the time step increases with mass $m_{\min }$ and decreases with $T$. The time step in tfMC is in most situations larger than the "standard" 1 fs time-step used in classical MD simulations. Although this is a promising result, it should be noted that the time-steps mentioned in Table I are still too small to allow accessing most of the long-time atomic events. However, tfMC combined with other acceleration techniques ${ }^{1,2}$ could potentially solve this problem.

As already pointed out in the introduction, the elegance of this approach lies in its simplicity: The proposed tfMC algorithm basically consists of five simple equations, namely Eqs. (11) and (23)-(26), that are fed by the initial positions and masses of the particles, their associated atomic forces, the temperature of the system, and a small-enough maximum displacement $\Delta$. While the positions, masses, and temperature are fixed input parameters, the atomic forces can be easily generated using either first-principles simulations or interatomic potentials. The definition of a boundary for a proper conservative atomic displacement is tackled in Sec. III A, where we will show that a $\Delta=0.10 \AA$ seems to be a conservative choice for many, if not most situations.

TABLE I. The time steps for different combinations of temperature and minimum mass (with $\Delta=0.12 \AA$ ).

\begin{tabular}{lcccccc}
\hline \hline & $T=200 \mathrm{~K}$ & $T=300 \mathrm{~K}$ & $T=400 \mathrm{~K}$ & $T=500 \mathrm{~K}$ & $T=600 \mathrm{~K}$ & $T=700 \mathrm{~K}$ \\
\hline$m_{\min }=1 \mathrm{u}$ & $\langle\Delta t\rangle=3.89 \mathrm{fs}$ & $\langle\Delta t\rangle=3.17 \mathrm{fs}$ & $\langle\Delta t\rangle=2.75 \mathrm{fs}$ & $\langle\Delta t\rangle=2.46 \mathrm{fs}$ & $\langle\Delta t\rangle=2.24 \mathrm{fs}$ & $\langle\Delta t\rangle=2.08 \mathrm{fs}$ \\
$m_{\min }=50 \mathrm{u}$ & $\langle\Delta t\rangle=27.5 \mathrm{fs}$ & $\langle\Delta t\rangle=22.4 \mathrm{fs}$ & $\langle\Delta t\rangle=19.4 \mathrm{fs}$ & $\langle\Delta t\rangle=17.4 \mathrm{fs}$ & $\langle\Delta t\rangle=15.9 \mathrm{fs}$ & $\langle\Delta t\rangle=14.7 \mathrm{fs}$ \\
$m_{\min }=100 \mathrm{u}$ & $\langle\Delta t\rangle=38.9 \mathrm{fs}$ & $\langle\Delta t\rangle=31.7 \mathrm{fs}$ & $\langle\Delta t\rangle=27.5 \mathrm{fs}$ & $\langle\Delta t\rangle=24.6 \mathrm{fs}$ & $\langle\Delta t\rangle=22.4 \mathrm{fs}$ & $\langle\Delta t\rangle=20.8 \mathrm{fs}$ \\
$m_{\min }=150 \mathrm{u}$ & $\langle\Delta t\rangle=47.6 \mathrm{fs}$ & $\langle\Delta t\rangle=38.9 \mathrm{fs}$ & $\langle\Delta t\rangle=33.7 \mathrm{fs}$ & $\langle\Delta t\rangle=30.1 \mathrm{fs}$ & $\langle\Delta t\rangle=27.5 \mathrm{fs}$ & $\langle\Delta t\rangle=25.4 \mathrm{fs}$ \\
$m_{\min }=200 \mathrm{u}$ & $\langle\Delta t\rangle=55.0 \mathrm{fs}$ & $\langle\Delta t\rangle=44.9 \mathrm{fs}$ & $\langle\Delta t\rangle=38.9 \mathrm{fs}$ & $\langle\Delta t\rangle=34.8 \mathrm{fs}$ & $\langle\Delta t\rangle=31.7 \mathrm{fs}$ & $\langle\Delta t\rangle=29.4 \mathrm{fs}$ \\
\hline \hline
\end{tabular}




\section{RESULTS AND DISCUSSION}

To test the tfMC method, and in particular the concept of time associated with this method, two cases have been studied. The first case in Sec. III A is a simple one-dimensional system. The main goal of this section is to acquire more insights in the approach. The second case in Sec. III B is more realistic and intends to illustrate the accuracy of the description obtained.

\section{A. Simple one-dimensional test case}

To gain some insights into the performance of the tfMC algorithms, we inspired ourselves with the work of Timonova et al. ${ }^{18}$ and considered the problem of diffusion in a simple one-dimensional sinusoidal potential surface $U(x)$,

$$
U(x)=\frac{Q}{2}\left[1-\cos \left(\frac{2 \pi x}{R}\right)\right],
$$

in which $Q$ and $R$ are the energy barrier and the period of the potential, respectively. In this potential the particle will mainly move in the valleys of the potential surface and irregularly cross a peak of the sinusoidal barrier to move to a neighboring valley. For this case, the rate $k$ at which these events happen can be calculated analytically using the transition state theory $(\mathrm{TST})^{21,22}$ and is equal to

$$
\begin{aligned}
k & =\sqrt{\frac{2 k_{B} T}{\pi m}}\left\langle\delta\left(|x|-\frac{R}{2}\right)\right\rangle \\
& =\sqrt{\frac{2 k_{B} T}{\pi m}} \frac{\exp \left[-Q /\left(k_{B} T\right)\right]}{\int_{-R / 2}^{R / 2} \exp \left[-U(x) /\left(k_{B} T\right)\right] d x},
\end{aligned}
$$

in which $\delta(x)$ is the Dirac delta function. The analytical formulation of the rate constant offers the possibility of rigorously comparing the simulated data to the theoretical ones.

We calculated the rate constant using two different methods. In the first one, the number of occurrences for which the particle crosses a peak of the sinusoidal potential is counted and divided by the total time The latter is simply defined as $N\langle\Delta t\rangle$ where $N$ is the number of MC steps and $\langle\Delta t\rangle$ is the averaged simulation time defined in Eq. (26). The other approach consists of calculating the diffusion coefficient via the Einstein relation

$$
D=\lim _{\Delta t \rightarrow+\infty} \frac{\left\langle\left[x\left(t_{0}+\Delta t\right)-x\left(t_{0}\right)\right]^{2}\right\rangle}{2 d \Delta t},
$$

with $d$ being the dimensionality of the system, equal to 1 in this case. The link with the rate constant $k$ is built using the relation

$$
k=\frac{2 d D}{(R / 2)^{2}} .
$$

Note that Eq. (30) contains a factor 2 in the denominator, which is not present in the original form of this equation. ${ }^{23}$ This additional factor is bound to the definition of the occurrence of a crossing event that has been used to describe the simulated particle. In a classical approach, when a particle crosses the top of the sinusoidal potential, it is accelerated downhill until it reaches the minimum of the potential basin. This is somewhat different from the behavior of the particle simulated by tfMC.

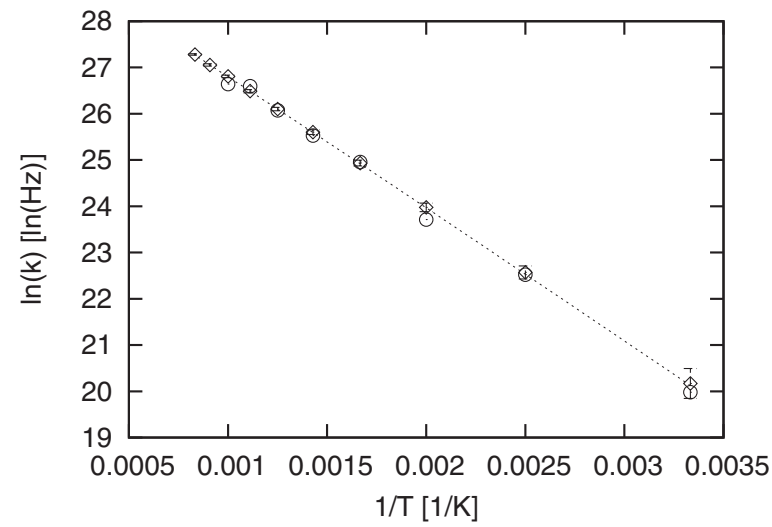

FIG. 3. The Arrhenius plot for the sinusoidal potential with $R=$ $1 \AA$ and $Q=0.25 \mathrm{eV}$. The dotted line represents the theoretical line obtained using Eq. (28) (TST). The diamonds are obtained by counting the number of jumps divided by time, while the circles are obtained by using Eqs. (29) and (30). The error bars around the diamonds are 1.96 times the obtained standard deviation.

Indeed, due to the probabilistic description, there is a nonzero probability that, although the simulated particle has crossed the maximum of the potential curve, it returns to its initial valley. If we define a jump as the occurrence of the particle crossing a maximum (consistent with the first method used), then the numbers of jumps of the real and simulated particles are in excellent agreement (see Fig. 3). The factor 2 present in the denominator of Eq. (30) accounts for a proper description of the jump length of the particle. Note that the agreement between the two techniques is of prime importance. In practice, the rate constant of atomic diffusion cannot be easily computed via the first approach, since it requires knowing the position of the transition state.

The tfMC algorithm has been tested for a sinusoidal potential with aa period $R=1 \AA$ and a barrier height $Q=$ $0.25 \mathrm{eV} . \Delta$ is chosen to be equal to $0.10 \AA$, the mass of the particle was taken equal to $60.0 \mathrm{u}$, and a temperature range from 300 to $1200 \mathrm{~K}$ has been considered. Each diamond point in Fig. 3 was obtained by averaging the results of 15 independent tfMC runs per temperature, each consisting of $10^{7}$ steps. The error bars where obtained by multiplying the standard deviation calculated for these $15 \mathrm{MC}$ runs by a factor of 1.96. When the 15 obtained data points have a normal distribution, these error bars stand for the $95 \%$ confidence interval. The circles were obtained via a single tfMC run consisting of $7.5 \times 10^{7}$ steps at each temperature. The diffusion coefficient in each of these runs is found by a linear fit of the average displacement squared as a function of $\Delta t$. The slope of this linear fit is taken equal to $2 D$ [see Eq. (29)]. The linear fit was carried out in the interval $\Delta t \in$ $\left[0.5 \times 10^{3}, 1.0 \times 10^{3}\right] \mathrm{fs}$; for smaller $\Delta t$ 's a parabolic line is observed, which reflects the ballistic transport. At higher $\Delta t$ 's, a deviation from the linear regime is obtained due to the finite simulation time. Figure 3 clearly illustrates that the results generated by the tfMC approach are in excellent agreement with the analytical ones, independent of the technique used. This suggests that our approach is valid for sufficient small $\Delta$.

In Sec. II B, we mentioned that the size of $\Delta$ and the curvature of the potential surface go hand in hand with the 


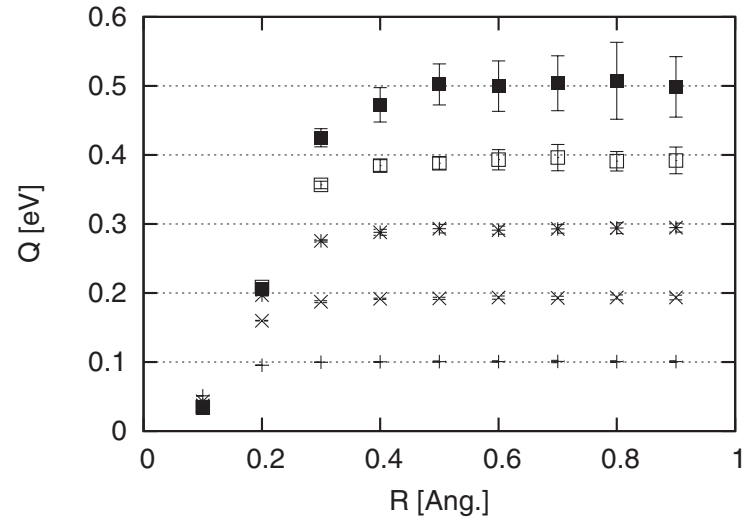

FIG. 4. Evolution of the computed $Q$ values with respect to the period $R$. The dashed line corresponds to the set-point chosen for $Q$.

accuracy of the description obtained by the ifMC approach. A potential surface having areas of large curvatures requires small $\Delta$, while in a rather smooth potential surface larger values of $\Delta$ are allowed. In order to gauge the impact of the curvature of the potential surface on $\Delta$, we have computed (see Fig. 4) the evolution of the computed $Q$ values with respect to the period $R$ as generated, by increasing both $R$ and $Q$ values with 0.1 per step in Eq. (27). At low values of $R$, the simulated value for $Q$ is significantly different from the set-point chosen for $Q$. From $R$ equal to $0.5 \AA$ onward, the match improves, reaching the $95 \%$ confidence interval for even the most varying surface $(R=0.5 \AA$ and $Q=0.5 \mathrm{eV})$. From this it seems that $\Delta=0.10 \AA$ is a rather robust tfMC step that is able to cope with strongly varying potential surfaces.

\section{B. Cu-adatom diffusion}

We benchmarked the results generated with the tfMC algorithm against the ones obtained with a classical MD approach in a three-dimensional system. Given the fact that the mechanisms that take place during the diffusion of $\mathrm{Cu}$ on a fcc copper (001) surface are well documented, ${ }^{24-26}$ they constitute as an ideal reference system. The model we used consists of a diffusing adatom on top of a copper (001) surface made of six consecutive layers of atoms (each layer contains 32 atoms) for a surface area of $14.5 \times 14.5 \AA$. The bottom layer is kept fixed during the simulation. The $\mathrm{Cu}-\mathrm{Cu}$ interaction is described by an embedded atom method (EAM) potential. ${ }^{27,28}$

The diffusion coefficients have been computed using Eq. (29) and have been converted to a rate constant via Eq. (30). Following TST, the rate constant takes the form

$$
k=v_{0} \exp \left(-\frac{E_{a}}{k_{B} T}\right),
$$

with $v_{0}$ the frequency factor and $E_{a}$ the activation energy. Note that, for the MD simulations, the factor two in the denominator of Eq. (30) has been excluded. The tfMC simulations were performed using $\Delta=0.10 \AA$, which corresponds to an averaged time step ranging from 7.8 to 10 fs per iteration for isothermal simulations, with a temperature evolving from 550 to $900 \mathrm{~K}$. In the low-temperature regime $(500-700 \mathrm{~K}), 20 \times 10^{6} \mathrm{tfMC}$

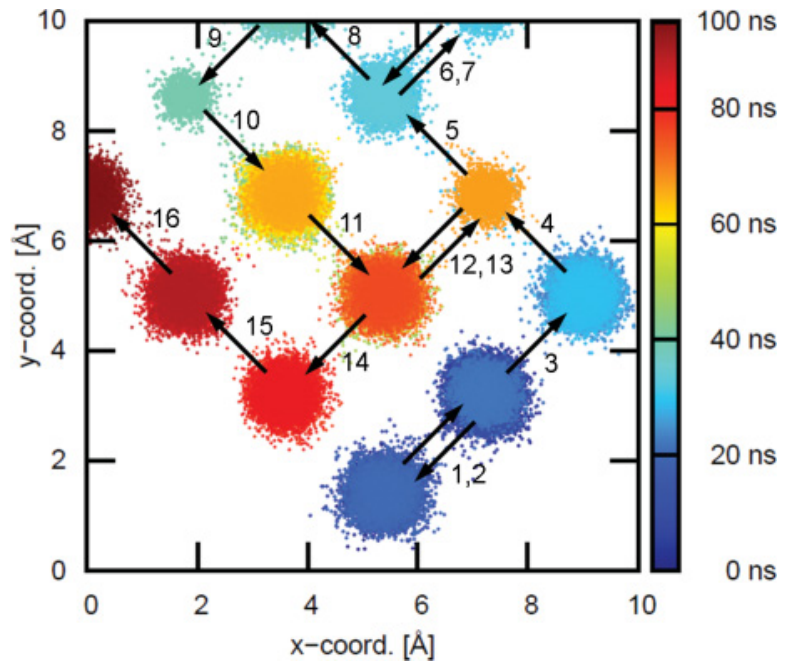

FIG. 5. (Color online) Illustration of the atomic movement of the $\mathrm{Cu}$ adatom on top of the $\mathrm{Cu}(001)$ surface with respect to time at $T=575 \mathrm{~K}$ with tfMC. The different colors represent the time course. The numbering illustrates the number of jumps undertaken by the $\mathrm{Cu}$ adatom.

steps were performed with a temperature interval of $25 \mathrm{~K}$ per simulation. At higher temperatures (750-900 K), $5 \times 10^{6}$ tfMC steps were carried out with a temperature increment of $50 \mathrm{~K}$. The MD simulations (using a Verlet algorithm ${ }^{29}$ and the Nosé thermostat ${ }^{30}$ ) use a time step of $1 \mathrm{fs}$ and took place in a temperature range of $650-900 \mathrm{~K}$ with a temperature increment of $50 \mathrm{~K}$. At each temperature $5 \times 10^{6}$ iterations where performed.

Figure 5 illustrates the dynamics of the adatom on the $\mathrm{Cu}(001)$ surface with tfMC. Interestingly, tfMC reproduces correctly the different mechanisms that occur during the diffusion process as observed in molecular dynamic simulations. ${ }^{26}$ Up to $\pm 630 \mathrm{~K}$ the adatom remains mainly trapped in a local potential well, and irregular jumps occur toward a neighboring potential well. At higher temperatures, an additional process takes place, i.e., an exchange mechanism occurs between the adatom and the underlying layer. The atom of the underlying layer that is expulsed through the exchange mechanism typically occupies a potential well close to the former adatom position. We also observe a concerted mechanism in which a new adatom pops out of the surface several angstroms away from the original potential well. This effect occurs when the original adatom inserts itself in the underlying surface layer

TABLE II. The activation barrier $E_{a}$ and frequency factor $v_{0}$ calculated in the present work and as reported in literature (Refs. 26 and 31-35).

\begin{tabular}{lcc}
\hline \hline Source & $E_{a}(\mathrm{eV})$ & $\nu_{0}(\mathrm{THz})$ \\
\hline Present work (MD) & 0.52 & 52.5 \\
Present work (tfMC) & 0.48 & 14.7 \\
Kong $(2008)^{31}$ & 0.51 & 35.8 \\
Yildirim $(2007)^{32}$ & 0.50 & 13.0 \\
Yildirim $(2006)^{33}$ & 0.51 & 7.5 \\
Kurpick $(2001)^{34}$ & 0.44 & 15.4 \\
Boisvert $(1997)^{35}$ & 0.49 & 20.0 \\
Evangelakis $(1996)^{26}$ & 0.43 & 20.9 \\
\hline \hline
\end{tabular}




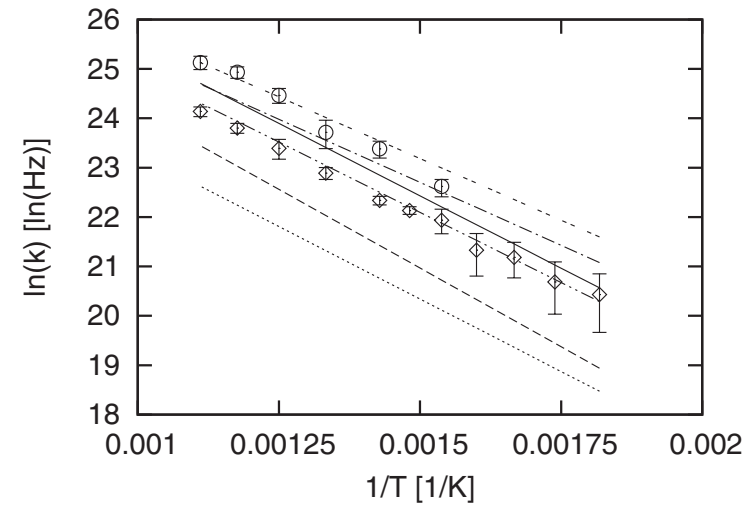

and pushes the neighborhood atoms sideways. As a result, the atoms in the layer exchange their positions until one of them is ejected toward the surface. ${ }^{26}$ Note that, because this chain process is fast, it contaminates the statistics used for the diffusion calculation of the adatom hopping. As a result, we eliminated these events from both the tfMC and the MD such that only the surface hopping mechanism is considered.

Figure 6 and Table II summarize the results obtained with the MD and tfMC methods together with recent literature results. $^{26,31-35}$ Note that the latter are obtained with a set of different $\mathrm{Cu}-\mathrm{Cu}$ interaction potentials and diffusion-coefficient calculation approaches. However, the work reported in Refs. 31-33, and 35 use the same formulation for the potential as the one used in this work. Further, it should be noted that the frequency-factor calculations are more sensitive to errors than the activation-energy ones, explaining the large spread in values reported in the literature.

The error bars of our results in Fig. 6 were obtained by calculating the $95 \%$ confidence interval, using a bootstrap technique $^{36}$ (no biasing or lack of symmetry was present in our data), for the mean-square displacement in Eq. (29). A relatively small difference is observed between the MD and tfMC computed data. Both lie within the distribution of the values reported in literature. The best possible fit for the MD Arrhenius plot leads to an energy barrier of $0.52 \mathrm{eV}$ and a frequency factor of $52.5 \mathrm{THz}$, with confidence intervals of [0.49, 0.56] eV and [45.9, 126] THz, respectively. The energy barrier and frequency factor obtained using the tfMC simulations are $0.48 \mathrm{eV}$ and $14.7 \mathrm{THz}$ with confidence intervals of [0.44, 0.51] eV and [7.80, 23.0] THz, respectively. The confidence intervals were obtained as the limiting values for a fitted line falling inside the intervals plotted in Fig. 6. Note that the calculated energy barriers of both simulations agree very well with each other and are in line with literature reports (see Table II). The computed frequency factor is significantly different for the MD and tfMC techniques, where the tfMC approaches provide values closer to the literature (see Table II).
FIG. 6. Logarithm of the rate constant $k$ plotted versus inverse temperature for the MD (circles) and tfMC (diamonds) simulations. Literature values (Refs. 26 and 31-35) are shown as straight lines in different formats according to the research work.

\section{CONCLUSIONS}

A new uniform-acceptance force-bias Monte Carlo method is developed based on the fundamental principles of a canonical ensemble. This approach leads to a further understanding of the fbMC methods without compromising the simplicity, inherent in this technique. The proposed method offers access to a statistically relevant time scale, allowing for a quantitative comparison with classical MD. As observed before, ${ }^{18}$ forcebias MC techniques are able to accelerate atomic events in comparison to classical MD simulations. In this work, we report that the time step taken per MC iteration is, in many cases, indeed larger than the "standard" 1 fs used for classical MD simulations, and mainly depends on the temperature of the system and on the mass of the lightest element.

The method has been successfully tested in a simple onedimensional sinusoidal potential. The simulated rate constant $k$ and diffusion coefficient $D$ gave excellent agreement with the analytical solution for sufficiently small atomic perturbation steps $(\Delta) . \Delta=0.10 \AA$ seems to be a relatively conservative perturbation step that could be used in most situations with atomic-size periodicity of the potential. A second reference system has been considered, in which the hopping of a copper adatom on top of a (100) copper surface has been studied using both MD and tfMC. The results of both simulations are in good agreement with each other and with values reported in literature. Only a small but significant difference is observed between the frequency factors of the MD and tfMC simulations.

The inclusion of a time scale, together with the appealing simplicity of the method and the enhancement of the time step compared to the classical MD approach, make this method very promising for materials-science simulations.

\section{ACKNOWLEDGMENTS}

E.C. Neyts acknowledges the FWO-Flanders (Fund for Scientific Research, Flanders) for financial support. This work is part of the SBO (Strategic Basic Research) MOFSHAPE project funded by the IWT (agency for Innovation by Science and Technology).

\footnotetext{
${ }^{3}$ A. F. Voter, J. Chem. Phys. 106, 4665 (1997).

${ }^{4}$ A. F. Voter, Phys. Rev. Lett. 78, 3908 (1997).

${ }^{5}$ A. F. Voter, Phys. Rev. B 57, 13985 (1998).

${ }^{6}$ M. R. Sorensen and A. F. Voter, J. Chem. Phys. 112, 9599 (2000).

${ }^{7}$ F. Montalenti and A. F. Voter, J. Chem. Phys. 116, 4819 (2002).
}

\footnotetext{
*pourtois@imec.be

${ }^{1}$ A. F. Voter, F. Montalenti, and T. C. Germann, Ann. Rev. Mater. Res. 32, 321 (2002).

${ }^{2}$ D. Perez, B. P. Uberuaga, Y. Shim, J. G. Amar, and A. F. Voter, Annu. Rep. Comp. Chem. 5, 79 (2009).
} 
${ }^{8}$ R. A. Miron and K. A. Fichthorn, J. Chem. Phys. 119, 6210 (2003).

${ }^{9}$ R. A. Miron and K. A. Fichthorn, Phys. Rev. Lett. 93, 128301 (2004).

${ }^{10}$ K. A. Fichthorn, R. A. Miron, Y. Wang, and Y. Tiwary, J. Phys. Condens. Matter 21, 084212 (2009).

${ }^{11}$ C. Pangali, M. Rao, and B. J. Berne, Chem. Phys. Lett. 55, 413 (1978).

${ }^{12}$ M. Rao, C. Pangali, and B. J. Berne, Mol. Phys. 37, 1773 (1979).

${ }^{13}$ S. Singh and S. Goldman, J. Comput. Phys. 62, 464 (1986).

${ }^{14}$ S. Goldman, J. Comput. Phys. 62, 441 (1986).

${ }^{15}$ G. Dereli, Mol. Simul. 8, 351 (1992).

${ }^{16}$ M. Rao and B. J. Berne, J. Chem. Phys. 71, 129 (1979).

${ }^{17}$ C. H. Grein, R. Benedek, and T. de la Rubia, Comput. Mater. Sci. 6, 123 (1996).

${ }^{18}$ M. Timonova, J. Groenewegen, and B. J. Thijsse, Phys. Rev. B 81, 144107 (2010).

${ }^{19}$ E. C. Neyts, Y. Shibuta, A. C. T. van Duin, and A. Bogaerts, ACS Nano 4, 6665 (2010).

${ }^{20}$ E. C. Neyts, A. C. T. van Duin, and A. Bogaerts, J. Amer. Chem. Soc. 133, 17225 (2011).

${ }^{21}$ R. Marcelin, Ann. Phys. 3, 120 (1915).
${ }^{22}$ J. B. Anderson, in Advances in Chemical Physics, Vol. 91 (John Wiley \& Sons Inc., New York, 1995), pp. 381-431.

${ }^{23} \mathrm{H}$. Mehrer, Diffusion in Solids: Fundamentals, Methods, Materials, Diffusion-Controlled Processes Springer Series in Solid State Science, Vol. 155 (Springer, Berlin, 2007).

${ }^{24}$ G. Antczak and G. Ehrlich, Surf. Sci. Rep. 62, 39 (2007).

${ }^{25}$ T. Ala-Nissila, R. Ferrando, and S. C. Ying, Adv. Phys. 51, 949 (2002).

${ }^{26}$ G. A. Evangelakis and N. I. Papanicolaou, Surf. Sci. 347, 376 (1996).

${ }^{27}$ M. S. Daw and M. I. Baskes, Phys. Rev. B 29, 6443 (1984).

${ }^{28}$ S. M. Foiles, M. I. Baskes, and M. S. Daw, Phys. Rev. B 33, 7983 (1986).

${ }^{29}$ L. Verlet, Phys. Rev. 159, 98 (1967).

${ }^{30}$ S. Nose, J. Chem. Phys. 81, 511 (1984).

${ }^{31}$ L. T. Kong and L. J. Lewis, Phys. Rev. B 77, 165422 (2008).

${ }^{32}$ H. Yildirim, A. Kara, and T. S. Rahman, Phys. Rev. B 76, 165421 (2007).

${ }^{33}$ H. Yildirim, A. Kara, S. Durukanoglu, and T. S. Rahman, Surf. Sci. 600, 484 (2006).

${ }^{34}$ U. Kurpick, Phys. Rev. B 64, 075418 (2001).

${ }^{35}$ G. Boisvert and L. J. Lewis, Phys. Rev. B 56, 7643 (1997).

${ }^{36}$ B. Efron, Ann. Stat. 7, 1 (1979). 